\title{
A hierarchical LSI-11 system for real-time control, interevent time storage, and data analysis
}

\author{
ALLAN F. DOYLE \\ Massachusetts Institute of Technology, Cambridge, Massachusetts 02139 \\ and \\ WILLIAM L. PALYA \\ Jacksonville State University, Jacksonville, Alabama 36265
}

\begin{abstract}
A general-purpose laboratory computer system using two Digital Equipment Corporation LSI-11s in a master-slave relationship is described. The implementation includes hardware to install an LSI-11 as a slave parallel processor, to provide special-purpose functions such as a real-time clock and a $4 \mathrm{~K}$ FIFO interprocessor buffer, and to establish a bus that permits the use of inexpensive interfaces to experimental equipment. The slave processor generates a complete $\log$ of each input or output event. Data in the form of the time of each event are continuously passed through the FIFO to a foreground task in the main LSI-11, which in turn stores the raw data on a hard disk. A macro library allows the easy development of experimentcontrol programs. A library of FORTRAN routines provides a pointer-based conditional and iterative search facility that can sift out any specifiable event or set of events from the data records for subsequent analysis. This computer system provides the user with an inexpensive facility to run up to 32 simultaneous experiments with complete data logging in foreground while program development, analysis, and word processing are concurrently being done in background.
\end{abstract}

The computer system described here provides the capability to dynamically analyze the results of animal research and a facility to easily conduct experimentation. Each input and output event and its time of occurrence is automatically stored. Subsequently, a pointer-based conditional and iterative search facility can be used to sift out any specifiable event or set of events from the data records. These selected data can then be analyzed as desired.

The present hierarchical dual processor configuration represents the current state of our laboratory system. It is composed of a main LSI-11 system for generalpurpose laboratory use and a subordinate, peripheral LSI-11 system dedicated to the control of experiments. The dual processor configuration allows general-purpose use while simultaneously running experiments.

A simpler single-processor system that provides for dynamic analysis is described by Palya and Doyle (1980). Unfortunately, under a very heavy research schedule, the simple system was inadequate; the computer could not be used for program development or data analysis while experiments were running. Although the RT-11 operating system has a foreground-background $(\mathrm{F} / \mathrm{B})$

The authors are greatly indebted to Elizabeth Palya for assistance in all phases of producing this system and manuscript. Requests for reprints, software, and schematics should be sent to William L. Palya, Department of Psychology, Jacksonville State University, Jacksonville, Alabama 36265. feature that processes low-priority tasks while real-time tasks are running, it could not be used because the worstcase 600-msec delay in switching from background to foreground would have been catastrophic.

\section{COMMERCIAL HARDWARE}

The main LSI-11 system is composed almost entirely of commercially available hardware that we assembled and configured as a system. Figure 1 provides a configuration diagram of the system. Backplanes, power supplies, and peripherals are mounted in three 19-in. bays. Plugged into an ADAC BP-1000 11-slot backplane with an ADAC PSC-1000 power-up sequencer are a KD11-F CPU board with 4K RAM and EIS/FIS, an additional 24K RAM, three DRV11 parallel interfaces, two DLV11 serial interfaces, and RXV11 dual floppy disk interface, a REV11-C ROM bootstrap, and an ACT Qbus-toUNIBUS transceiver called a Univerter. On the UNIBUS are a Plessey PMC-11 disk controller and a Western Peripherals TC-130 tape controller. A RX01 dual floppy disk system, two Wangco 10-Mbyte cartridge disk drives, and two Wangco tape drives provide mass storage. The system console is a Lear Siegler ADM-3 CRT. A 6800 microprocessor functions as an intelligent channel controller for a Centronics 761 serial printer, a Houston Instrument DP-7 drum plotter, and a Megatek BP-734 graphics controller driving a TRW vector display.

The peripheral LSI-11 system, although mostly 


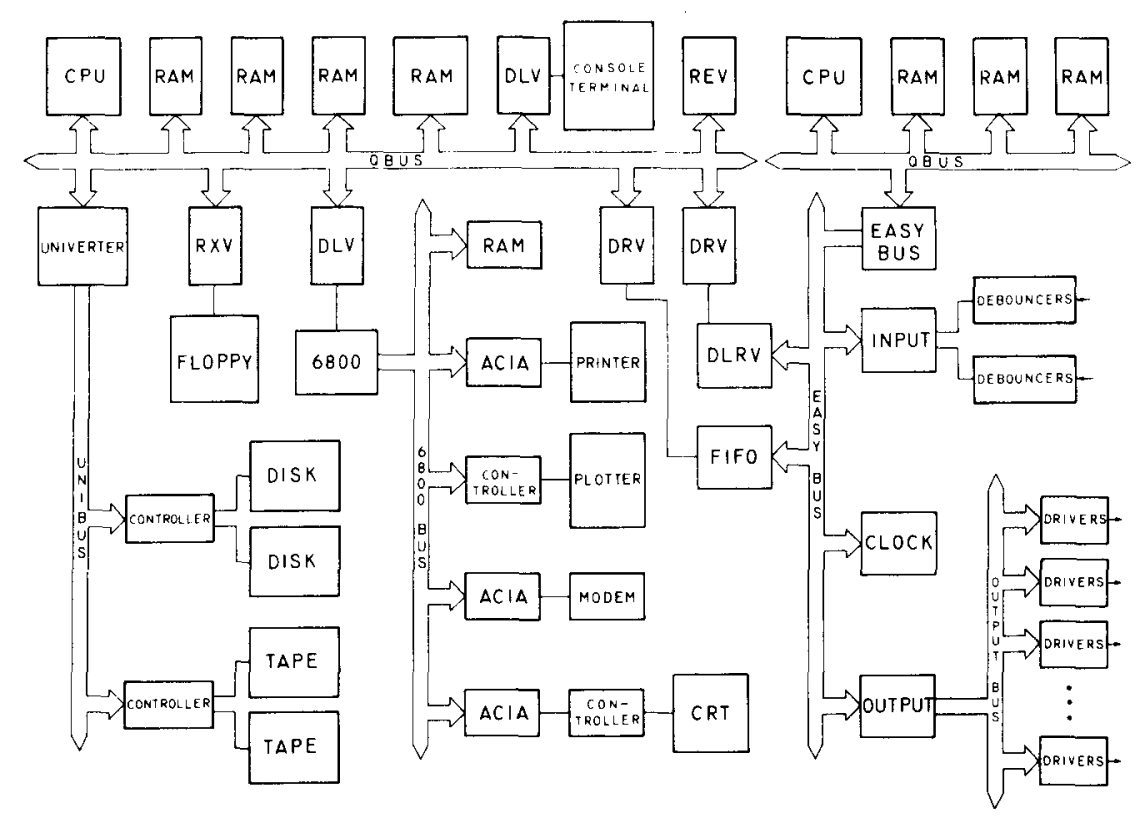

Figure 1. Block diagram of the hierarchical LSI-11 system.

custom designed, includes a KD11-F with its 4K RAM and an additional $12 \mathrm{~K}$ RAM plugged into the DEC PDP-11/03 enclosure with its four-slot backplane and H780 power supply.

\section{CUSTOM HARDWARE}

A successful hierarchical system requires fast efficient communication between the peripheral processor and the main system, the hardware implementation of several special functions, and an inexpensive way of interfacing new functions to the system. These requirements were met by designing a simple bus structure called the Easy Bus. Presently, two interprocessor communication modules, a real-time clock, a queueing input system, and a modular output system plug into this bus.

\section{Easy Bus}

Interfacing to the Qbus is expensive and relatively complex because it has data and address information multiplexed over the same set of wires. The main advantages offered by the Easy Bus are separate address and data lines, addresses that are only 8 bits wide, and the ability to use inexpensive boards and connectors.

The bus translator board plugs into the Qbus of the peripheral LSI-11 and translates between Qbus protocol and Easy Bus protocol. The Easy Bus supplants the top 128 locations of memory in the peripheral processor, which includes the DEC-supported address for the console terminal. Figure 2 provides a block diagram of the bus translator board. Electrically, the Easy Bus is the same as the Qbus, with open-collector, active-low, terminated lines of $120-\mathrm{ohm}$ impedance.

The Easy Bus is jumpered to its own backplane. Labbuilt function modules can be plugged into the backplane and are effectively on the Qbus of the peripheral LSI-11. The CPU cannot distinguish Easy Bus from Qbus boards, except that the Easy Bus cannot handle direct memory access.

\section{DLRV11}

Computers load initial programs with a loader program called a bootstrap. The bootstrap is loaded in one of three ways: beforehand as a permanent program stored in ROM, such as in the REV11-C; manually through a switch register; or manually through the console terminal with a special utility program for that purpose stored in ROM. This special utility program, ODT in the LSI11, essentially converts the console terminal into a switch register. ODT is part of the LSI-11 microcode and allows the user to open various memory locations and deposit a program that bootstraps the system.

Developing a special-purpose ROM board or purchas-

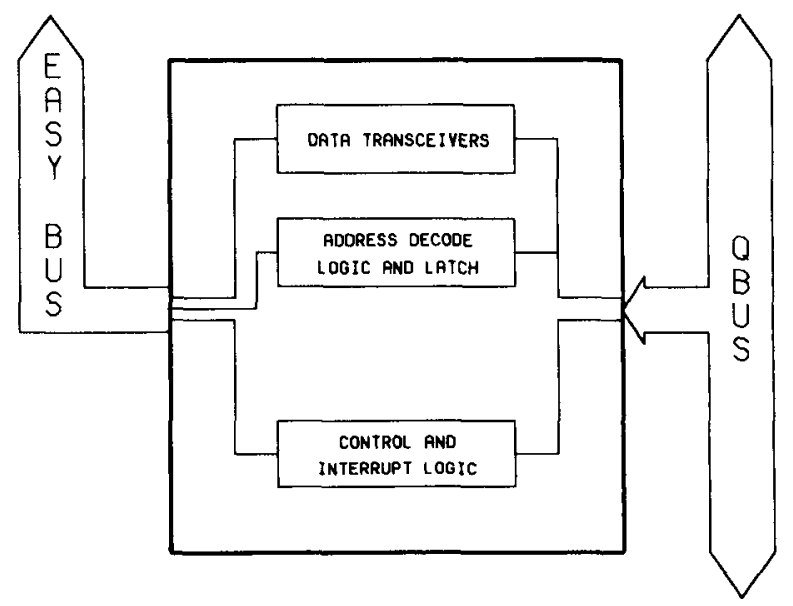

Figure 2. Block diagram of the bus translator board. 


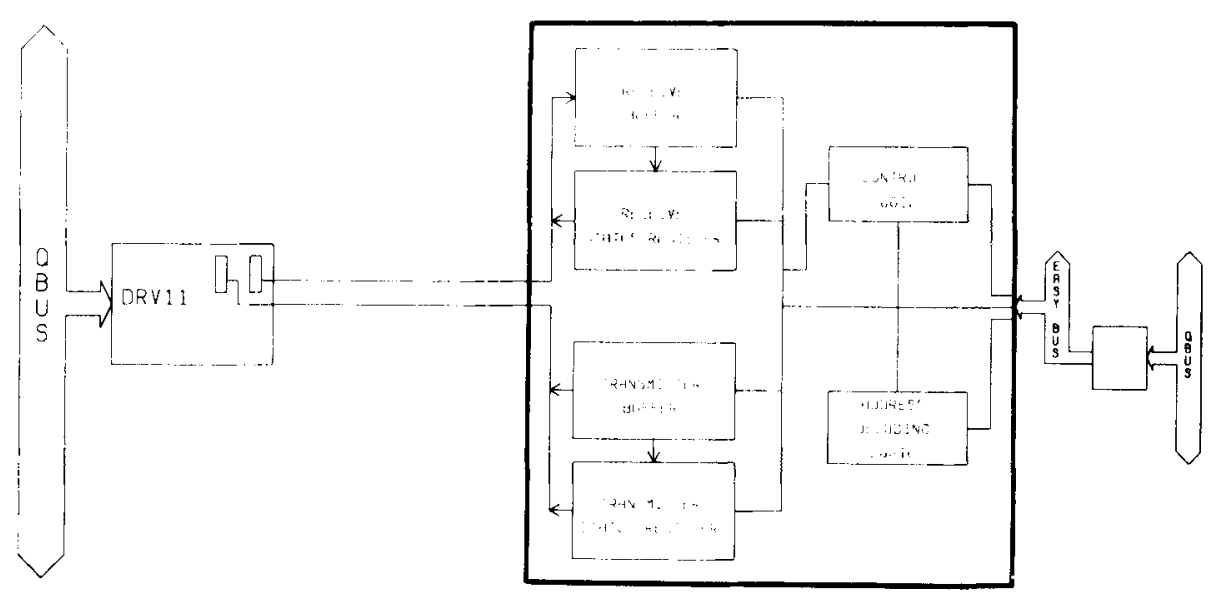

Figure 3. Block diagram of the DLRV 11 board.

ing the REV11-C ROM was not cost effective considering the ODT facility already available in the peripheral computer.

ODT is designed to communicate with a serial board. Therefore, the obvious, although not best, solution would have been to plug DLV11 serial boards into the main computer and into the peripheral computer and pass the commands necessary to boot up the peripheral system through this serial link from the main computer to the peripheral computer. This communication would have been limited to $19.2 \mathrm{~K}$ bits/ $\mathrm{sec}$ and would have been unacceptably slow once the peripheral system was up and running. Clearly, 16-bit parallel communication was more desirable.

The solution was to use a parallel communication link that could emulate a DLV11 serial unit as far as the peripheral computer's ODT is concerned. This DLRV11 is a 16-bit parallel interface that can provide the control information needed by ODT. Figure 3 provides a block diagram of the DLRV11 board. The main LSI-11 communicates with the DLRV11 in parallel through a DRV1 1 parallel unit. To load a bootstrap into the peripheral system, the main system sends ODT commands 1 byte at a time through its DRV11 to the peripheral computer's DLRV11 and responds appropriately to responses issued by the peripheral computer's ODT. The bootstrap that is loaded into the peripheral system, in turn, efficiently loads 16 -bit words through the same channel. Once the peripheral system is no longer in ODT mode and is running the 16-bit-wide loader, it can utilize the full word width and full speed of the interface. The two systems can then communicate at about $100 \mathrm{~K}$ words/sec, which is 80 times faster than two serial DLV11s.

\section{FIFO}

The peripheral system could have sent data back to the main system continuously. It would then have been necessary for the main computer to be almost totally occupied with being ready to pass data to the disk. This would not have solved the original problem of freeing computer time for general use. Alternatively, the peripheral system could have saved blocks of data in its memory and then sent data to the main computer a block at a time. This would have incurred a heavy overhead penalty, since each datum would have to be handled twice by the peripheral computer-once to store it and once to send it to the main system.

In the scheme that is used, the peripheral system handles data only once. Data are written into a memory location that is the upper port of a $4 \mathrm{~K}$ first-in first-out (FIFO) buffer. These data are passed to the main system in the order they are written into the upper port of the FIFO. The data are available to the main processor 450 nsec after being stored by the peripheral processor. This FIFO buffer provides "full" and "empty" status information to both processors and can be accessed by both processors simultaneously without arbitration delay.

The FIFO plugs into the peripheral computer's Easy Bus and communicates to the main computer's second DRV11 board. Figure 4 is a block diagram of the FIFO card.

\section{CLOCK}

To relieve the peripheral CPU from the burden of software timekeeping, a 32-bit clock was implemented on the Easy Bus. This clock provides time since the current session began in two consecutive memory locations. A $60-\mathrm{Hz}$ square wave from the ADAC PSC1000 is used by the clock as a time base. The clock rate can be anything up to about $1 \mathrm{MHz}$, but the $60-\mathrm{Hz}$ signal allows standard DEC time-handling software to be used during later data analysis. Figure 5 provides a block diagram of the clock board.

\section{OUTPUTS}

The present system provides 240 high-current outputs. The outputs are organized into 30 8-bit bytes. Each of the 240 outputs is individually accessible 


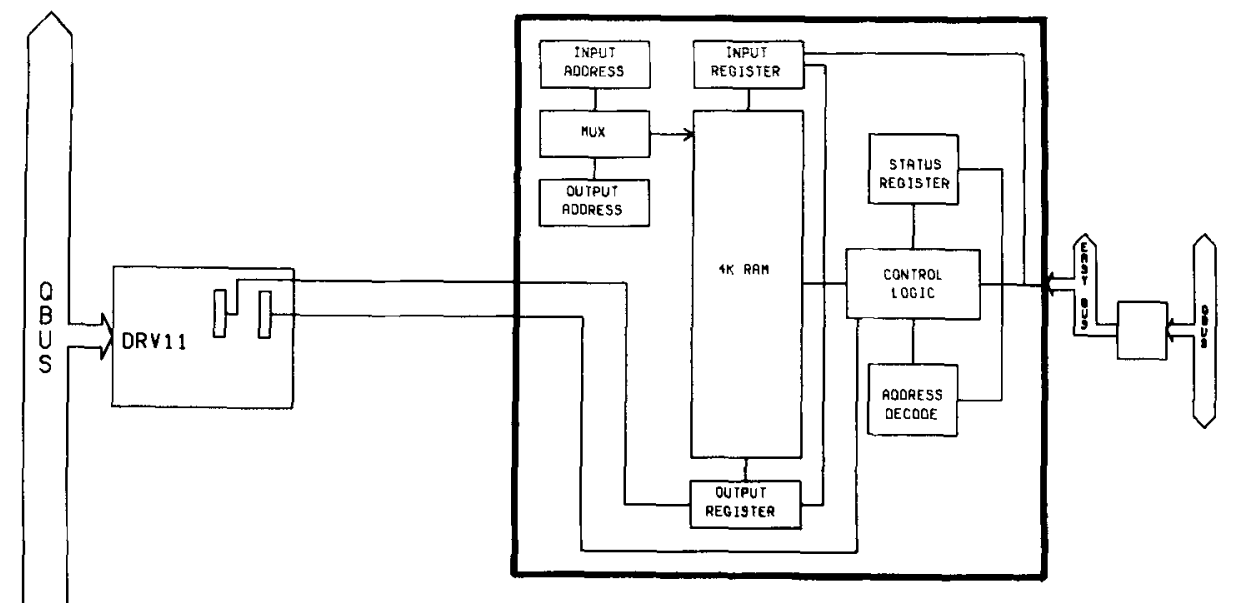

Figure 4. Block diagram of the FIFO card.

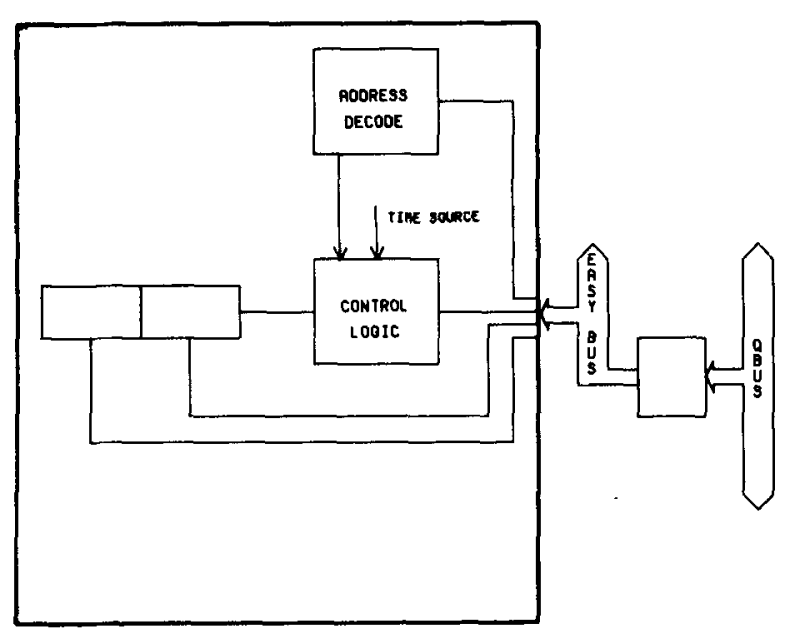

Figure 5. Block diagram of the clock board.

through the same Easy Bus address. The upper half of the 16-bit memory location specifies the address of the output byte; the lower 8 bits represent the information to be written to that byte. The outputs are latched and drive Sprague UHP 407 500-mA 40-V drivers.

The output interface consists of the controller, that decodes which byte is being addressed, and the latchdriver units. The controller is on an Easy Bus card; the latch-driver units plug into their own "output" bus implemented on a separate backplane. Each of 10 small circuit boards contains drivers and LED indicators for 3 bytes. Figure 6 provides a block diagram of the controller board and a latch-driver board.

\section{INPUTS}

It takes a great deal of software overhead to debounce contact closures and to decode which input needs attention. An input controller board that plugs into the Easy Bus controls two input interfacing boards. Each decodes and debounces 32 inputs in hardware.
Each input has a multivibrator that blocks out any bounces for a selected time period following each response. Different bounce characteristics can be accurately screened by installing different resistor-capacitor networks. Input queueing is also done in hardware. Each input is assigned a code. When one or more inputs are activated, the logic causes an interrupt. The input hardware then successively passes the codes of each active input to the input register until all inputs have been serviced. The processor is assured that all active inputs and only active inputs have been obtained by successively reading the input register until the interrupt flag is cleared by the input hardware. Figure 7 illustrates a block diagram of the input cards.

\section{COMMERCIAL SOFTWARE}

The main system runs DEC's RT-11 operating system. RT-11 provides housekeeping, utilities, major languages, and a F/B feature. In a F/B monitor the background is permitted to be active whenever the foreground is not active or when the foreground is waiting for some event, such as the completion of an I/O operation. The present hierarchical system uses a foreground task to assure interptocessor communication whenever it is required, and it concurrently permits background tasks to be performed whenever data are not being passed between machines. This architecture makes very efficient use of system resources.

All experiment-control programs are written in MACRO. It is an extremely powerful assembler that produces efficient and fast code. A MACRO library is presently being built that will make it relatively simple for a researcher to write MACRO programs to control experiments. These programs are almost as straightforward as those written in CLOC (Palya \& Doyle, 1980).

Analysis programs are written in FORTRAN. A library of FORTRAN subroutines that is modeled after EXPLOR (Palya \& Doyle, 1980) is being developed. 


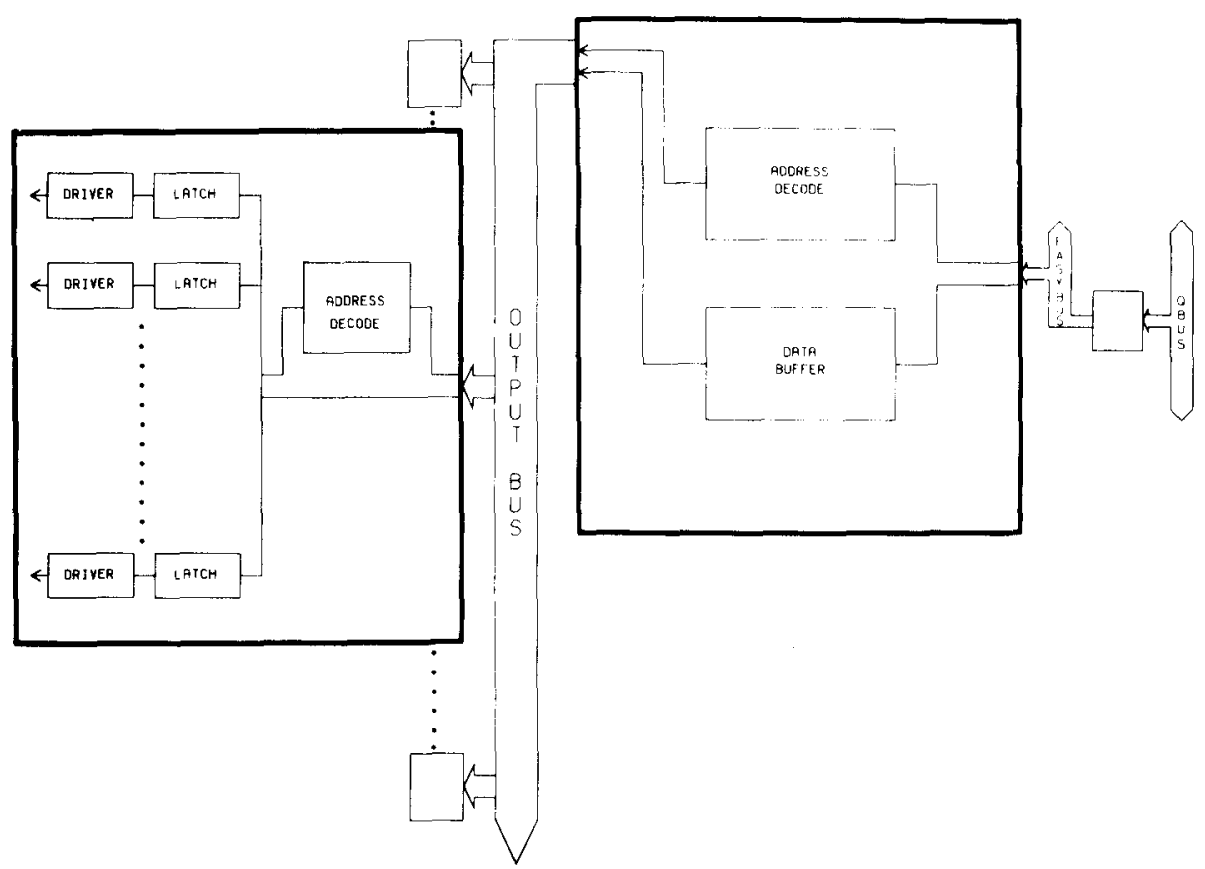

Figure 6. Block diagram of the controller board and a latch-driver board.

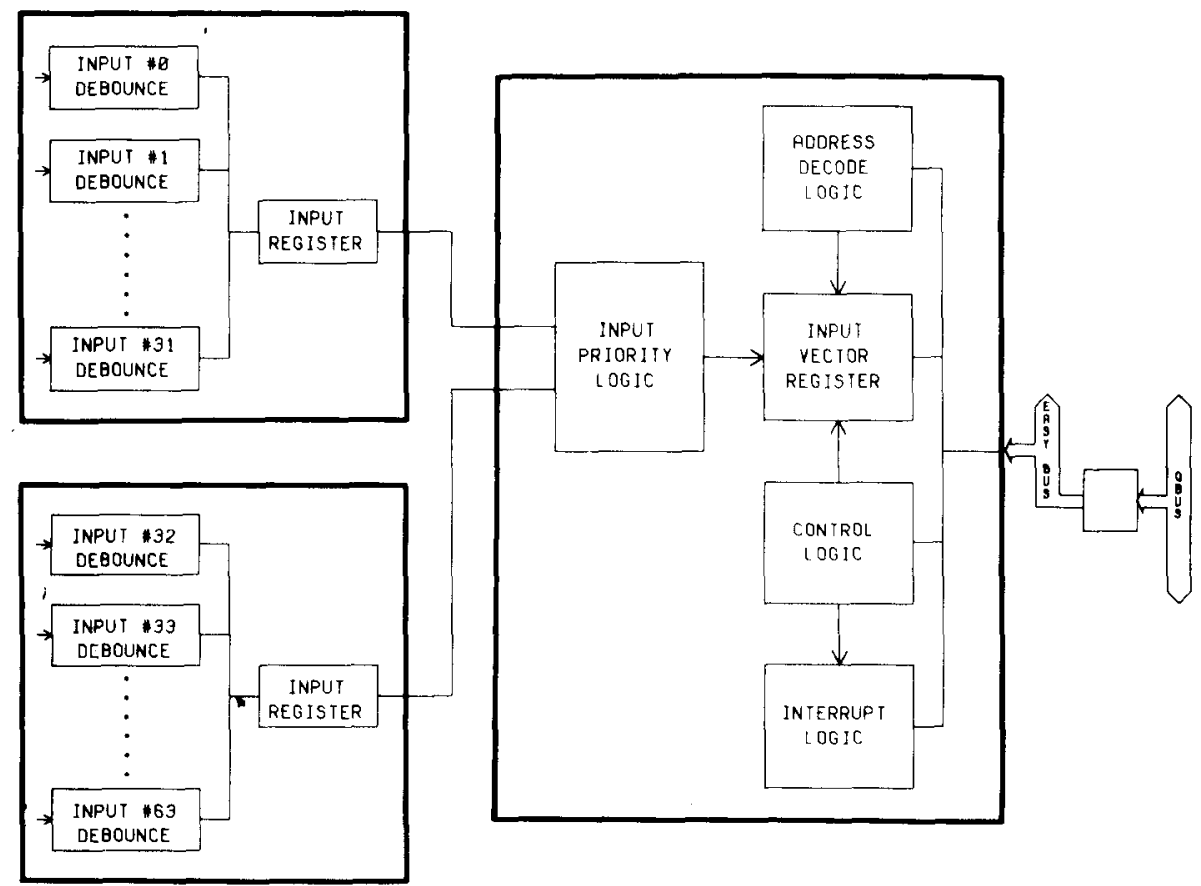

Figure 7. Block diagram of an input card.

\section{CUSTOM SOFTWARE}

Individual control programs for each experiment, written in MACRO with library macros, are assembled and then linked with the multiprocessing software. The resulting run module is down-line loaded to the periph- eral processor by the foreground task. This foreground task is responsible for all interprocessor communication, which also includes passing the raw data from the peripheral processor to the disk. When the data are to be analyzed, a utility program separates the data of one subject from the combined data files stored on the disk. 
A library of FORTRAN subroutines allows specific subsets of the data to be conveniently obtained from data files.

\section{Interprocessor Communication: BRUNV3}

BRUNV3 is written in FORTRAN and is responsible for the interprocessor communication. This foreground task is used to down-line load experiment-control programs and to pass data to the disk from the peripheral processor; the background is free for such tasks as developing new programs, data analysis, or text editing.

BRUNV3 is run as a foreground task. It first opens the data file on the disk and then asks for the name of the run module to be down-line loaded to the peripheral processor. Once in the peripheral processor, the run module is started. Using the DLRV11 and BRUNV3, the peripheral processor requests from the console terminal the subject number and the number of reinforcers for each of the experiment-control programs. BRUNV3 is then instructed to "go to sleep" by the peripheral processor.

BRUNV3 "wakes up" every $100 \mathrm{msec}$ thereafter, empties the FIFO of any new data produced by the peripheral processor, and checks the DLRV11 for messages such as one indicating that all control programs have run to completion. If this message occurs, BRUNV3 empties the FIFO one last time and closes the data file on the disk into which it has been dumping all data. It then informs the console terminal that the run has been successfully terminated.

Table 1 illustrates the user-system dialogue for BRUNV3. The user types FRUN BRUNV3/N:1000 on the terminal. The FRUN specifies that the program is to be run as a foreground task. Memory must be reserved when executing a foreground job. The $/ \mathrm{N}: 1000$

Table 1

BRUNV3 System-User Dialogue

. FRUN BRUNV3/N:1000

F>

DATA. BV $3=$ PROG . SAV

$\uparrow F$

TR64, BV3=HRUN1, SAV

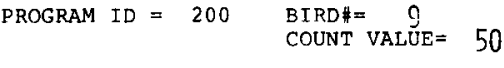

PROGRAM ID $=300 \quad$ BIRD\# $=89$

COUNT VALUE $=60$

$\begin{array}{ll}\text { PROGRAM ID }=500 & \begin{array}{l}\text { BIRD\# }=4 \\ \text { COUNT VALUE }=35\end{array}\end{array}$

- - - - BRUNV3 BIRDS RUNNING

$\uparrow \mathrm{B}$

$B>$

$\uparrow C$

(Any Background task)

Note-User input is in large type. reserves 1,000 words of memory. Following F>, the system prefix for all messages from the foreground task, the foreground task requests the file name of the output data file and then the file name of the experimentcontrol program. To direct keyboard input to the foreground job, the user enters a control $F(\uparrow F)$. The user then types the data file name, TR64. BV3, an equal sign, and then the experiment-control program, HRUN1. SAV. The peripheral processor then requests the subject number and the number of reinforcers for each experimentcontrol program. After the last entry the foreground job indicates that the subjects are running. The user can then type a control $B(\uparrow B)$ that directs all keyboard input to the background job. A B $>$ is returned, which indicates that subsequent messages are from the background job. Any subsequent control $\mathrm{C}(\uparrow \mathrm{C})$ invokes the background monitor. The user may then use all the facilities of RT-11. When all subjects are finished, the foreground task interrupts with $\mathrm{F}>$ and prints its message that the subjects are finished.

BRUNV3 is active for an average of $10 \mathrm{msec} / 100 \mathrm{msec}$, which provides the background $90 \%$ of the main CPU's time. A further reduction in overhead is obtained when the interprocessor communication is interrupt driven.

\section{Multiprocessing Software}

The software necessary to implement multiprocessing is called as a subroutine by each experiment-control program. This subroutine stores the next consecutive instruction address on the stack, effects a context switch to the next experiment-control program, and then initiates program flow at the appropriate instruction for that program. In this way, each control program can be written independently without regard to the 31 other programs that can be run concurrently. To the researcher, each control program is written as if it runs in isolation and runs continuously. The subroutine calls are built into the macros that the researcher strings together to construct an experiment-control program.

When first loaded, the peripheral processor obtains the subject number and reinforcer count for each control program. Then the first control program is started. The first program executes one of the macros available in the macro library, such as SET. The SET macro turns on a bit, dumps the accompanying data into the FIFO, and then issues a call to the context-switching subroutine. The subroutine terminates the currently executing program and stores the next consecutive instruction address. The software invoked by the call then takes the contents of all of the registers and saves them in a table. It then gets the register contents for the experimentcontrol program to be executed next from the context table and loads them into the registers. Finally, the multiprocessing handler places the next instruction address of the program to be executed on the stack and issues a return from subroutine instruction. The processor takes the first word on the stack and places it into 
Table 2

Sample Header Program for Five Programs

- TITLE HEADER FOR RUN1 .MCALL INIT, START, GO

$\begin{array}{ll}\text { RUN } 1:: & \text { INIT \#5 } \\ & \text { START PROGI } \\ & \text { START PROG2 } \\ & \text { START PROG3 } \\ & \text { START PROG4 } \\ & \text { START PROG5 } \\ & \text { GO }\end{array}$

. END RUN1

the program counter. The program counter therefore begins execution at the appropriate instruction in the designated program.

The "header" program provides the skeleton of the run module, which is down-line loaded and performs several initialization functions. It allocates a space for each control program to be inserted at link time and when running in the peripheral processor. It sets up the multiprocessing context tables for the appropriate number of control programs, obtains the required numbers and reinforcer counts, and then starts the control programs. Header is written by stringing together macros contained in the macro library and is then assembled using the MACRO assembler.

Table 2 illustrates a sample header program for running five separate programs. TITLE is the running head for assembly listings. MCALL specifies which macros are used in the program. The RUN1:: label is the name of the program and indicates where execution is to begin. INIT \# is the module that sets up the context tables in the peripheral processor. The $\# 5$ in the example specifies that five programs will be included in this run. The START PROGX lines hold a position for the experimentcontrol programs that are added at link time. The GO macro effects the initialization and start-up sequence after the run module is loaded into the peripheral LSI-1 1 . The .END RUN1 statement terminates assembly and specifies that the program is to be autostart.

The individual control programs and the header program are linked with the RT-11 utility LINKER using the BV3OBJ library. The resulting run module is stored on the disk and is loaded into the peripheral processor by BRUNV3 each time it is to be used.

\section{Experiment-Control Macro Library: BV3MAC-BV30BJ}

Control programs are written by stringing together macro calls to the BRUNV3 macro and object library following MACRO assembler syntax. This library was developed to allow the researcher to put together complex control programs with only a limited knowledge of assembly language and the custom hardware. All macros log data, pass data to the FIFO, and context switch automatically. The SET instruction, for example, turns on a bit in the interface, passes the program ID number, the bit affected, and the time since the program started to the FIFO data stream, and then initiates the context switch to the next control program.

The researcher writes each control program separately using the MACRO assembler. The current macro library for experiment control is presented in Appendix A.

Table 3 provides a sample program for running a fixed-ratio 100 (FR100) schedule. The control program starts at TRIAL:. The preceding lines of code are overhead or initialization. The .TITLE line indicates the running head for assembly listings. .MCALL specifies the macros to be used by the program. BLKW is used to reserve 100 words of memory so that the program can maintain necessary information through context switches. The FR100:: and .WORD line provides the global identifier and the program ID for this specific program. INPUT \#31. indicates to the input hardware that input \#31 is to be assigned to this program. SET \#1, \#100 activates the particular bit that turns on the power in the box. The label TRIAL: provides a symbolic address for the beginning of a loop that will be executed over and over. SET \#2, \#20 turns on a white light behind the center key. The next instruction COUNT \#100, \#31. waits until 100 pecks have been counted on Key 31. CLEAR \#2, \#20 turns off the center key light. SET \#2, \#1 raises the food magazine and operates the magazine light. TIME \#240. waits 240 ticks, or $4 \mathrm{sec}$. CLEAR \#2, \#1 turns reinforcement off. ENDTST \#1, \#10 decrements the number of reinforcers remaining and determines if all reinforcers have been provided for that session. If the correct number of reinforcers has been given, the program is terminated. If the correct number of reinforcers has not been given, JMP TRIAL returns program flow to TRIAL:.

Figure 8 illustrates the overall process of running an experiment with the present hierarchical system. When

Table 3

Source Program for an FR100

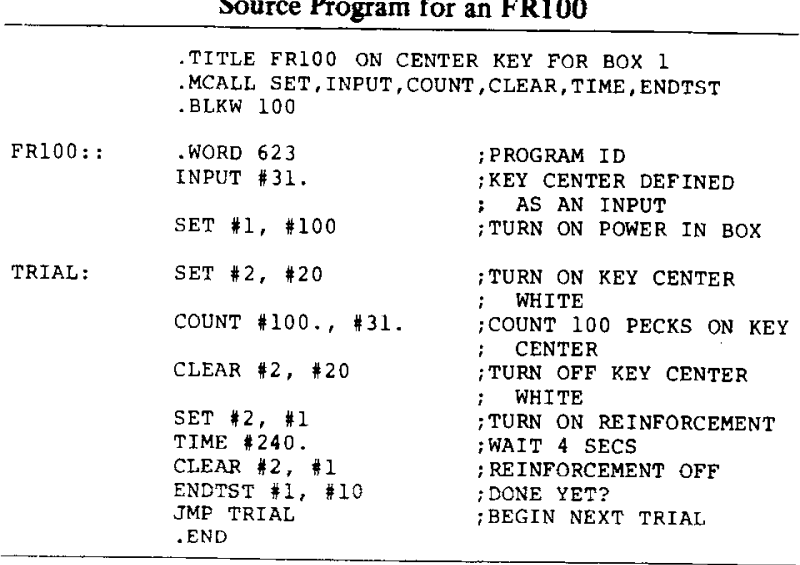




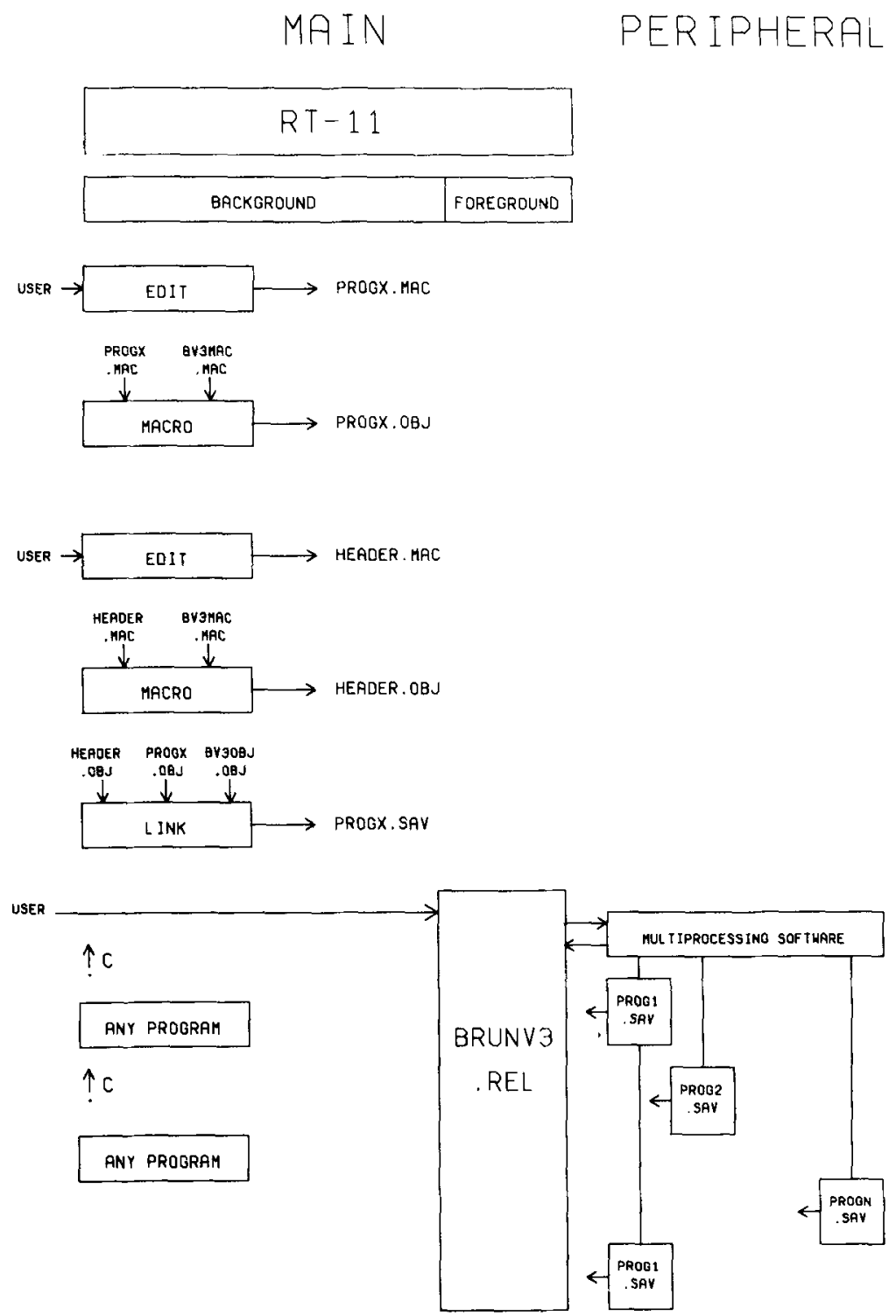

Figure 8.

in the background of the RT-11 operating system, the user runs the EDITOR and creates an experiment-control program in MACRO called, for example, PROG.MAC. Using the MACRO assembler, PROG.MAC is assembled with the BV3MAC.MAC macro library. This assembly process produces an object module called, for example, PROG.OBJ. This process is repeated for each experimentcontrol program. The user then runs the EDITOR again and sets up a header that specifies the control programs to be run at one time. This program is a string of macros needed to set up the multiprocessing software for the number of programs that will run concurrently. This program is called HEADER.MAC and is assembled using the BV3MAC.MAC library. This assembly produces the object module called HEADER.OBJ. The LINKER is then used to link control programs and their header with the BV3OBJ.OBJ library. This linking process produces the executable program for down-line loading called, for example, PROGX.SAV. Whenever experiments are to be conducted, the interprocessor communication software, BRUNV3, is run in foreground. BRUNV3 is used to down-line load the desired run module into the peripheral processor and pass data returned from it to the hard disk. This enables the user to execute background jobs while experiments are running.

\section{SPLV3}

BRUNV3 does not maintain separate data files on the mass storage device for each of its concurrently running experiments. The data from all of the chambers in any 
Table 4

SPLV3 User-System Dialogue

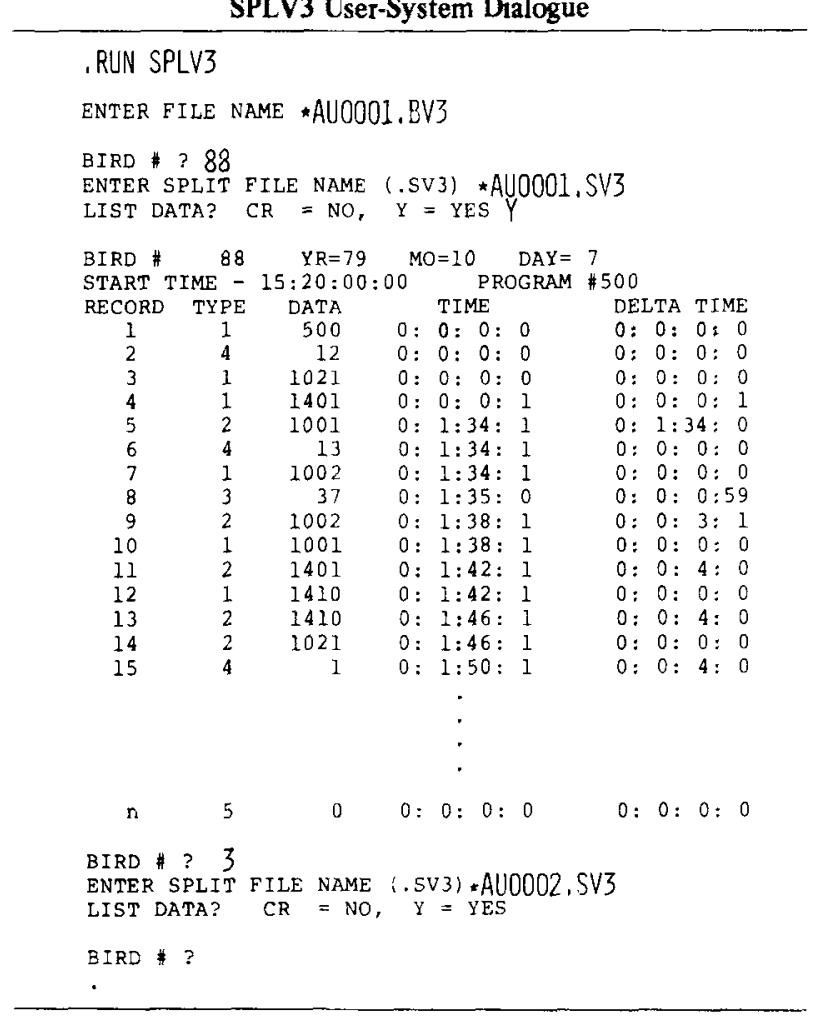

Note-User input is in large type.

Table 5

Sample XPLRV3 Program

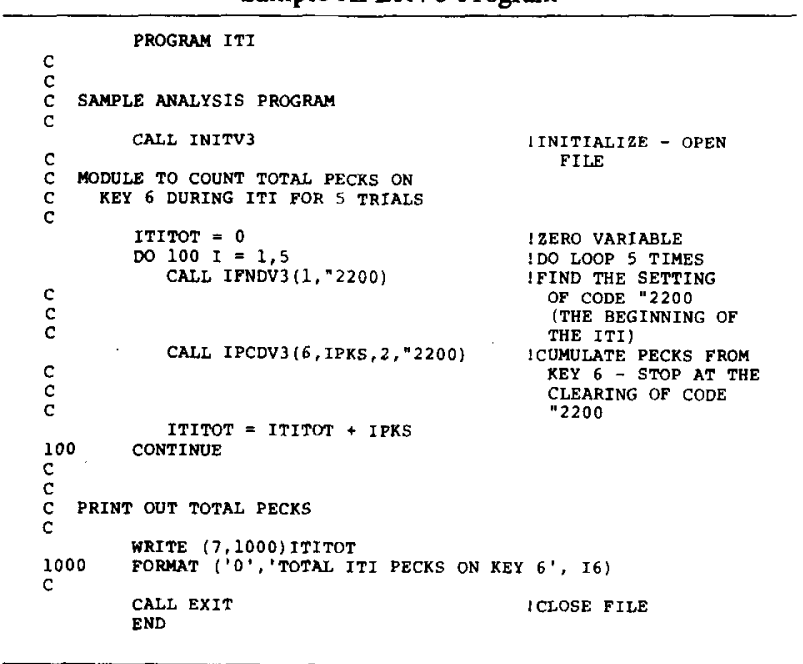

one run are mixed into the same data file. Before analysis can take place, therefore, the data from the experiment of interest must be separated from the mixed data file. This is accomplished with a small utility program called SPLV3. The resulting data file is a two-dimensional array of numbers. Each consecutive row represents a consecutive event. The first column in each row is the data type, the second column contains the actual data, and the third column is the cumulative time coded into standard DEC format with $60-\mathrm{Hz}$ resolution. When hard copy of the data is requested, two additional columns are appended for user convenience. An initial column is added to each row to indicate the record number, and a final column is calculated that indicates the time since the last event, or "delta" time. These two additional columns are added on printouts and are not provided to the disk file created by SPLV3.

Table 4 illustrates the SPLV3 user-system interaction. SPLV3 first requests the name of the mixed data file that contains the data of interest. It then determines which data are to be split out and the name for the output file. It then asks whether or not data are to be listed on the printer. If so, it prints the header information, followed by a time-coded event-by-event list of everything that happened in that box on that session; if not, it simply creates a disk file with the data of that subject.

\section{XPLRV3}

A FORTRAN subroutine library named XPLRV3 provides a convenient pointer-based conditional and iterative search facility for interevent time data. A researcher can dynamically explore data by simply moving a pointer through the data file while extracting any specifiable event or set of events. The complete subroutine library of XPLRV3 modules is presented in Appendix B.

A sample XPLRV3 program is provided in Table 5. The first instruction, CALL INITV3, is a subroutine call that requests from the console terminal what data file is to be analyzed and establishes a link to the data file. ITITOT $=0$ resets the value of the ITITOT counter. The DO and CONTINUE statements bracket a loop. Every. thing in this loop is done five times. CALL IFNDV3 moves the pointer forward until it encounters the beginning of an intertrial interval (ITI), which is code 1, "2200. The CALL IPCDV3 subroutine moves the pointer forward, counting keypecks until the ITI ends. It cumulates the number of pecks encountered in the ITI in the temporary variable named IPKS. The ITITOT $=$ ITITOT + IPKS instruction is a running total of the ITI pecks. The WRITE and FORMAT instructions output the contents of the ITITOT counter after typing "TOTAL ITI PECKS ON KEY 6" to the console terminal. The CALL EXIT and END instructions terminate the program.

\section{REFERENCE}

Palya, W. L., \& Doyle, A. F. A simple LSI-11 system for realtime control, interevent time storage, and data analysis. Behavior Research Methods \& r.witimentation, 1980, 12, 210-220. 
Appendix A

Macro Library

All numbers are octal unless followed by a decimal point. ; denotes that a comment follows.

All conventions in the MACRO-11 assembler language apply. Any MACRO instructions can be used.

\section{OUTPUT INSTRUCTIONS}

$$
\text { SET \#__, \#__ \# }
$$

These instructions turn on (set) or turn off (clear) an output. SET \#0, \#1 turns on Byte 0, Bit 1. SET R2, R3 turns on the bit specified in R3 in the byte specified in R2.

\section{MARKER \#}

This instruction has no effect other than to put a marker in the data file. It enables XPLRV3 to more conveniently move to a point in time that has no other event change. There are up to 65,000 different markers. MARKER \#15. puts decimal 15 into the data stream from that box. MARKER R4 puts the contents of R4 into the data stream from that box as a marker.

\section{INPUT \#}

There are 0.63 inputs. They must be defined in the beginning of a program, for example, INPUT $\# 0$ or INPUT \#6.

COUNT \# , \#-

This macro counts a specified number of pecks on one input before executing the next instruction in the control program. COUNT $\# 5, \# 3$ counts five pecks on Input 3.

\section{ANYINP \#XXXXXX, \#YYYYYY}

This instruction sets up a multipath branch depending upon which of several inputs occur, and it requires some additional program overhead. The tables that specify the input alternatives to be considered and which path is to be associated with each potential input must occur somewhere in the program. $\mathrm{XXXXXX}$ is any label of the potential inputs.

\section{XXXXXX: .WORD 11 \\ WORD 12 \\ .WORD 13.}

YYYYYY is any label of an array of symbolic addresses that identify the destination of the possible branches.

$$
\begin{array}{ll}
\text { YYYYYY: } & \text { WORD AAAAAA } \\
& \text {.WORD BBBBBB } \\
& \text {.WORD CCCCCC }
\end{array}
$$

\section{UTILITIES}

\section{RANDOM}

This macro puts a random number into Register R0.

TIME \#

This macro waits a specified amount of time in ticks $(60$ ticks $=$ $1 \mathrm{sec})$ before executing the next instruction in the control program. The limit is 32,760 ticks in one macro. Longer delays can be implemented with more than one time instruction. TIME \#120. waits $2 \mathrm{sec}$.

ENDTST \#_, \#

This decrements the end-of-session count value entered at the beginning of a session in BRUNV3. Each time an ENDTST is executed, the count value is decreased by 1 until it is 0 , which terminates the program and shuts down the chamber. The numbers used in the instruction specify the byte and bit of the power relay for that box.

STOP

This unconditionally stops the program. This can be used instead of ENDTST in conjunction with some other session-termination scheme.

.END

This must be the last line in each program.

\begin{tabular}{|c|c|c|c|c|}
\hline Record 1 & $\begin{array}{l}\text { Bird No. } \\
\text { Low time start } \\
\text { Data type } \\
1 \text { (SET) } \\
2 \text { (CLEAR) } \\
3 \text { (INPUT) } \\
4 \text { (MARKER) }\end{array}$ & $\begin{array}{l}\text { Year } \\
\text { High time start } \\
\text { Data } \\
\text { byte-mask } \\
\text { byte-mask } \\
\text { input \# } \\
\text { marker \# }\end{array}$ & $\begin{array}{l}\text { Month } \\
\text { Program ID } \\
\text { low time } \\
\text { low time } \\
\text { low time } \\
\text { low time } \\
\text { low time }\end{array}$ & $\begin{array}{l}\text { Day } \\
\text { Local ID } \\
\text { high time } \\
\text { high time } \\
\text { high time } \\
\text { high time } \\
\text { high time }\end{array}$ \\
\hline
\end{tabular}

\section{XPLRV3}

Data file record format:

Time is a two-element array, STIM (2) is low time and STIM(1) is high time. CALL CVTTIM (STIM, IH, IM, IS, IT) converts a record to hours, minutes, seconds, and ticks.

\section{MODULES}

When the following modules are invoked as functions rather than as subroutines, they return +1 normally and return -1 if an end of file or beginning of file is encountered. XPLRV3 uses Logical Unit 2 for disk I/O; therefore, user programs must not use this logical unit.

\section{PROGRAM OVERHEAD}

\section{CALL INITV3}

This or IPRHV3 must be the first statement in the program (other than comments and the PROGRAM statement). It opens files and reads the header information.

\section{CALL IPRHV3 (IDEVIS)}

This can substitute for INITV3 in the program. It does the same thing as INITV3 except that it prints the header information on the console device.

\section{CALL EXIT}

This must be the next-to-the-last statement in the program. It closes files.

\section{END}

This must be the last statement in the program.

\section{POINTER MOVEMENT}

\section{CALL BEGNV3}

This module moves the pointer to the beginning of the data file and spaces past the header so that the first data record is immediately ahead of the pointer.

$$
\begin{aligned}
& \text { CALL IRDV3 (J, K, IH, IM, IS, IT) } \\
& \text { I = IRDV3 (J, K, IH, IM, IS, IT) }
\end{aligned}
$$

This module reads the next data record and returns: $J$ the data 
type, $\mathrm{K}$ the data, IH the hour, IM the minute, IS the second, and IT the tick.

$$
\begin{aligned}
& \text { CALL IFNDV3 (ITYP, IDATA) } \\
& I=\text { IFNDV3 (ITYP, IDATA) }
\end{aligned}
$$

This module moves the pointer to a datum specified by ITYP (the data type) and IDATA (the data).

$$
\begin{aligned}
& \text { CALL IPCDV3 (KEY, IPK, ITYP, IDATA) } \\
& \text { I = IPCDV3 (KEY, IPK, ITYP, IDATA) }
\end{aligned}
$$

This module cumulates pecks into IPK from the key specified by "KEY" and stops when it gets to the datum specified by ITYP (the data type) and IDATA (the data).

$$
\begin{aligned}
& \text { CALL NPCDV3 (JKEY, JPK, ITYP, IDATA, N) } \\
& I=\quad \text { NPCDV3 (JKEY, JPK, ITYP, IDATA, N) }
\end{aligned}
$$

This module cumulates pecks on any set of keys up to the datum specified by ITYP (the data type) and IDATA (the data).

$\mathrm{JKEY}=\operatorname{array}(\mathrm{N})$ array of the key numbers to be monitored $J P K=$ array $(N)$ counters for each of the specified keys $\mathrm{N}=$ array size, i.e., number of keys to be monitored

CALL IPTMV3 (INPA, INPB, INPC, IH, IM, IS, IT, IPEKSA, IPEKSB, IPEKSC)

$I=\quad$ IPTMV3 (INPA, INPB, INPC, IH, IM, IS, IT, IPEKSA, IPEKSB, IPEKSC)

This module cumulates the pecks on the specified inputs that occur within the time specified by IH (hours), IM (minutes), IS (seconds), and IT (ticks).

INPA = input number of first key

INPB $=$ input number of second key

INPC = input number of third key

IPEKSA = total number of pecks counted on Input $A$ in that time

IPEKSB $=$ total number of pecks counted on Input $B$ in that time

IPEKSC $=$ total number of pecks counted on Input $C$ in that time

$$
\begin{aligned}
& \text { CALL ITMSV3 (IH, IM, IS, IT, JH, JM, JS, JT) } \\
& \text { I }=\text { ITMSV3 (IH, IM, IS, IT, JH, JM, JS, JT) }
\end{aligned}
$$

This module computes the time change between two records by subtracting IH, IM, IS, IT from JH, JM, JS, JT. I positive or zero is a normal return; if I is negative, an overflow or underflow occurred.

$$
\begin{aligned}
& \text { CALL ITMAV3 (IH, IM, IS, IT, JH, JM, JS, JT) } \\
& \text { I = ITMAV3 (IH, IM, IS, IT, JH, JM, JS, JT) }
\end{aligned}
$$

This module adds IH, IM, IS, IT to JH, JM, JS, JT. I positive or negative is a normal return; if $I$ is negative, an overflow occurred.

$$
\begin{aligned}
& \text { CALL JTMAV3 (IH, IM, IS, IT, JH, JM, JS, JT, ISUMTM) } \\
& \text { I = JTMAV3 (IH, IM, IS, IT, JH, JM, JS, JT, ISUMTM) }
\end{aligned}
$$

This module adds IH, IM, IS, IT to JH, JM, JS, JT and returns an unconverted sum time, ISUMTM, in integer * 4 format. This module allows the addition of several times. $I=0$ is a normal return; $I=-1$ indicates that an error occurred.

CALL JTMSV3 (IH, IM, IS, IT, JH, JM, JS, JT, IDIFTM)

$$
\mathrm{I}=\mathrm{JTMSV} 3 \text { (IH, IM, IS, IT, JH, JM, JS, JT, IDIFTM) }
$$

This module subtracts IH, IM, IS, IT from JH, JM, JS, JT and returns an unconverted difference time, IDIFTM, in integer * 4 format. This module allows the subtraction of several times. $I=0$ is a normal return; $I=-1$ indicates that an error occurred.

CALL IBINV3 (IBEGA, IBEGB, IBEGC, INPA, INPB, INPC, IH, IM, IS, IT, IBINS, ITBINA, ITBINB, ITBINC)

$I=$ IBINV3 (IBEGA, IBEGB, IBEGC, INPA, INPB, INPC, IH, IM, IS, IT, IBNS, ITBINA, ITBINB, ITBINC)

This module sorts the pecks that occur during a specified time period (such as a trial) into a specified number of bins. If a $10-\mathrm{sec}$ trial were divided into five bins, all the pecks of the first 2 sec would be added into the first bin, the pecks in the next $2 \mathrm{sec}$ in to the next bin, and so on.

IBEGA = the type code to begin first bin

IBEGB = the data code to begin first bin

INPA = input number of first key

INPB = input number of second key

INPC = input number of third key

IH, IM, IS, IT = the duration of the block over which pecks are to be binned

IBINS = the number of bins into which that duration is to be partitioned

ITBINA = array IBINS long that provides total pecks in Key A in that period

ITBINB = array IBINS long that provides total pecks in Key B in that period

ITBINC = array IBINS long that provides total pecks in Key C in that period 